\title{
The Origin and Nature of Neptune-like Planets Orbiting Close to Solar Type Stars
}

\author{
Adrián Brunini and Rodolfo G. Cionco ${ }^{1}$ \\ Facultad de Ciencias Astronómicas y Geofísicas de la Universidad Nacional \\ de La Plata, Paseo del Bosque s/n, La Plata, Argentina. Instituto de As- \\ trofísica de La Plata (IALP), CONICET.
}

Number of pages: 27

Number of figures: 3

Number of tables: 1

\footnotetext{
${ }^{1}$ Also at Universidad Tecnológica Nacional-Facultad Regional San Nicolás, Colón 332, 2900, San Nicolás, Argentina.
} 
Propposed running head: Origin of Neptune-like extrasolar planets

Correspondence should be directed to: Adrián Brunini. Facultad de Ciencias Astronómicas. Paseo del Bosque s/n La Plata (1900) Argentina. Phone: +54-221-4236593/4- Fax: +54-221-423-6591. E-mail; abrunini@fcaglp.unlp.edu.ar 


\begin{abstract}
The sample of known exoplanets is strongly biased to masses larger than the ones of the giant gaseous planets of the solar system. Recently, the discovery of two extrasolar planets of considerably lower masses around the nearby stars GJ 436 and $\rho$ Cancri was reported. They are like our outermost icy giants, Uranus and Neptune, but in contrast, these new planets are orbiting at only some hundredth of the Earth-Sun distance from their host stars, raising several new questions about their origin and constitution. Here we report numerical simulations of planetary accretion that show, for the first time through N-body integrations that the formation of compact systems of Neptune-like planets close to the hosts stars could be a common by-product of planetary formation. We found a regime of planetary accretion, in which orbital migration accumulates protoplanets in a narrow region around the inner edge of the nebula, where they collide each other giving rise to Neptune-like planets. Our results suggest that, if a protoplanetary solar environment is common in the galaxy, the discovery of a vast population of this sort of 'hot cores' should be expected in the near future.
\end{abstract}


Key words: Giant planets - Origin - Extrasolar planets. 


\section{Introduction}

The most plausible hypothesis regarding the formation of giant planets is furnished by the core accretion model (Mizuno, 1980), where a solid core grows by accretion of planetesimals (Safronov, 1969). When the core mass increases above a certain critical value (10-15 Earth masses), a violent accretion of nebular gas onto the solid core starts, leading to the formation of a gaseous giant planet (Pollack et al., 1996). The final mass of the gaseous envelope depends on several factors, including the amount of gas present in the primordial disk, and the ability of the solid core to reach the critical mass before the dissipation of the surrounding nebula.

Two extrasolar planets of low mass were recently discovered orbiting around the nearby stars GJ 436 (Butler et al., 2004) and $\rho$ Cancri (McArthur et al., 2004). They are like Neptune in mass, but these new planets are orbiting at only $0.02 \mathrm{AU}$ and $0.04 \mathrm{AU}$ respectively from their host stars.

Standard disk models show that at the distances where the new planets have been found (Butler et al., 2004; McArthur et al., 2004), the temperature is $600-2000 \mathrm{~K}$ (depending on the stellar type). Although at these distances, the existence of solid material cannot be ruled out, the temperature is too 
hot as to expect a substantial amount of it as to form large solid cores there. More likely, giant planets like these ones form at much larger distances from the star, and subsequently they migrate inwards. Theory and numerical simulations have shown that growing protoplanets can experience orbital migration, traveling very far from their birth place (Goldreich and Tremaine, 1980; Lin and Papaloizou, 1986; Ward 1986; Ward 1997a). In fact, planetary migration is a natural consequence of the tidal interaction with the nebula. The most plausible mode of migration for protoplanets is inward in a quasi circular orbit (Ward, 1986; Ward, 1988; Ward, 1997a; Tanaka et al., 2002; Tanaka and Ward, 2004). The tidal interaction with the star or the truncation of the disk by the stellar magnetosphere, prevent the planets to fall onto the star. Planetary migration have been invoked to explain close stellar companions (Lin et al., 1996) of large mass ('hot Jupiters'). Ward (1997b), have also discussed in situ formation of ice giants planets by migration of embryos to the inner protoplanetary disk limit. Besides, Ida and Lin (2004) have delimited conditions for which ice giants could form in a variety of circumstellar disks. Laughlin et al. ( 2004a), show that whereas ice giants form readily around stars of all masses, Jupiter-like planets are difficult to form around the smallest stars. The new ice planets discovered support the 
findings of Laughlin et al. (2004a); indeed, GJ436 is a M dwarf type star, but in the $\rho$ Cancri system (which is a 'solar' G8V star) are coexisting the new 'Neptune' with three other Jupiter-like planets. However, N-body simulations of planetary formation showing how Neptune-like planets as the ones recently discovered could form, have not been yet carried out. In this paper, we report the first numerical simulations of planetary accretion, showing that the formation of Neptune-like planets very near the central star could be a common by product of planet formation, in a standard nebular environment.

\section{Simulations}

We have performed a series of N-body numerical simulations of the accretion of solid cores, that include, for the first time, the dynamical effects of the gaseous environment on them, as predicted by the more recent results of the theory of planet-disk interaction (Ward, 1997a; Tanaka et al., 2002; Tanaka and Ward, 2004). In the set of simulations reported here, we have explored a model whose initial mass is of the order of the mass expected in the minimummass nebular model (NM) (Hayashi et al., 1985).

For the accretion of solid cores, we follow the most plausible model which 
is based on the concept of 'oligarchic' growth (Kokubo and Ida, 1998; Kokubo and Ida, 2000). In this model, only few large objects growth at almost comparable rates, separated by amounts determined by their masses and distances from the star. The gravitational scattering with the largest protoplanets dominates the dynamical evolution of the background planetesimals, which for this reason cease to growth. However, the planetesimal disk, although representing only a fraction of the total solid mass, may contribute to the further growth of migrating protoplanets. We have performed four numerical simulations where 100 small protoplanets of 0.5 Earth masses were initially placed on nearly circular, very low-inclination orbits around a star of one solar mass $\left(M_{*}\right)$. They were spread from $5 \mathrm{AU}$ to $15 \mathrm{AU}$, their mutual separation were generated at random, but following a $r^{-1.5}$ profile.

It is very difficult to handle the amount of solid material required for a NM $\left(\sim 70 M_{\oplus}\right)$-note that we must integrate bodies spread over an extended disk-; thus, we are compelled to make some approximations. Oligarchyc growth models predict (at an initial time) a variety of embryo masses at different heliocentric distances, but we assume a uni-modal embryonic mass distribution. The number and mass of the embryos cited above, are plausible initial conditions in an protoplanetary disk model of a few minimum-masses 
(see, Thommes et al., 2003). Although the time-forming of such embryos can be very large, even in a several NM model, if the random velocities of the embryos are sufficiently damped, the formation of big bodies can be expected (Thommes et al., 2003). We make the hypothesis that the tidal interaction with a NM gaseous disk (not considered in oligarchyc growth studies) can produce substantial velocity damping and radial mobility of big embrios as to strength the growth process (see e.g., Tanaka and Ida, 1999). Besides, during the epoch of the formation of these embryos the nebular environment could have been denser, helping to the growth process. Nevertheless, we think that detailed time and mass dependence of the initial conditions may not be important to a bottom-up set of simulations.

In addition, a swarm of residual planetesimals was included, occupying the same space than the protoplanets. To make the simulations numerically tractable, we used 200 planetesimals of 0.1 Earth masses, distributed with a surface density proportional to $r^{-1.5}$. Although the planetesimals interact with the protoplanets, we ignored the self-gravity between them (see e.g., Cionco and Brunini, 2002). A gaseous disk consistent with the minimum mass solar nebula model (total mass, density and temperature distribution, and scale height) was considered (Hayashi et al., 1995). On the planetary 
embryos, the disk acts dynamically on the orbital semimajor axes and eccentricities, as prescribed by the theory of density waves. The gaseous disk was assumed truncated at $0.1 \mathrm{AU}$ and gradually dissipates at a constant rate, in such a way that it completely disappears after $10^{7} \mathrm{yr}$, as observations of circumstellar disks around young stars indicates (Beckwith and Sargent, 1996). The gaseous disk also affects the orbits of the planetesimals through aerodynamic gas drag (Adachi et al., 1976), but as if their radii were of 1000 $\mathrm{km}$.

To carry out these simulations, we have adapted our N-Body hybrid code (Brunini and Melita, 2002; Cionco and Brunini, 2002) to include all these effects. Our model of planet-disk interaction includes migration of type I.

We didn't follow the 'classical' approach of summing the corresponding component torque over each active resonance (see e.g., Cionco and Brunini, 2002); here, we follow the most efficient strategy to be applied either in the leap-frog part of the hybrid integrator as in the Bulirsch-Stoer numerical scheme used in it, in order to prescribe the orbital evolution predicted by density waves theory. We correct the embryo velocities through a Stokes non-conservative force, yielding the same semi-major axis $(a)$ and eccentricity $(e)$ evolution predicted by density waves up to first order in $e$, applied in a 
consistent fashion, taking into account the disk properties at the different zones reached by migrating bodies.

This force, acting on a protoplanet of mass $M_{p}$ and mean motion $\Omega$, produces an acceleration $\left(A_{S}\right)$ and must be applied following the gas-planet relative velocity direction $\left(\breve{v}_{r e l}\right)$ as in the case of aerodynamic drag (Adachi et al., 1976):

$$
A_{S}=K M_{p} \rho c_{s}^{\alpha} V_{r e l}
$$

where $K$ is a parameter; $\rho$ is the volumetric gaseous density; $c_{s}$ is the sound speed in the medium (assumed as gas temperature dependent); $V_{\text {rel }}$ is the modulus of the relative gas-planet velocity. In what follows, we assume that all the distance-dependent quantities are evaluated at the semi-major axis of the perturber.

Adachi et al. (1976), have found expressions for the mean variation of the orbital elements over one orbital period due to Eq. (1). Rewriting $\rho \propto \Sigma / h$, being $\Sigma$ the gaseous surface density, and $h$ the isothermal vertical scale height defined by: $h \propto c_{s} / \Omega$, we can write up to the first order in $e$ : 


$$
\begin{gathered}
\frac{1}{a}\left\langle\frac{d a}{d t}\right\rangle \propto-K M_{p} \Sigma \Omega c_{s}^{\alpha-1}(h / a)^{2} \\
\frac{1}{e}\left\langle\frac{d e}{d t}\right\rangle \propto-K M_{p} \Sigma \Omega c_{s}^{\alpha-1}
\end{gathered}
$$

The perturbing acceleration -Eq. (1)-, must reproduce the same evolution of semi-major axis and eccentricity estimated by density waves. This estimates are: (see e.g., Ward, 1986; Ward, 1988; Ward, 1997a; Tanaka et al., 2002; Tanaka and Ward, 2004):

$$
\begin{aligned}
& \frac{1}{a} \frac{d a}{d t} \propto-\frac{M_{p}}{M_{*}}\left(\frac{\sum a^{2}}{M_{*}}\right) \Omega(a / h)^{2}, \\
& \frac{1}{e} \frac{d e}{d t} \propto-\frac{M_{p}}{M_{*}}\left(\frac{\Sigma a^{2}}{M_{*}}\right) \Omega(a / h)^{4} .
\end{aligned}
$$

Expressions (2)-(3) and (4)-(5) are equivalent adopting $\alpha=-3$, and $K \propto$ $G^{2}$, being $G=\Omega^{2} a^{3} / M_{*}$, the gravitational constant.

Finally, we adopted an appropriated constant in the definition of the parameter $K$ in order to match 'exactly' the semi-major axis and eccentricity evolution result predicted by Eqs.(4)-(5) in our simulated disk. 
In our simulations, a $1 M_{\oplus}$ planet migrating at $5 \mathrm{AU}$, reach the inner disk limit in $\sim 1 \times 10^{6} \mathrm{yr}$, and the time required for the same body, to reach a circular orbit (i.e., in attain $e \sim 5 \times 10^{-5}$ ), is of $\sim 5 \times 10^{4} \mathrm{yr}$.

The inner edge of the disk is an uncertain parameter, nevertheless, the migration can be halted by gas depletion near 0.1 AU (see Ida and Lin, 2004, and references therein); thus, we assume that a protoplanet reaching the 'real' disk limit, can go on migrating until its outer Lindblad resonances get out of the disk by a factor of $\simeq 2$ respect to the physical disk limit. Then, according to the frictional approach, when a protoplanet reaches $0.05 \mathrm{AU}$, it is assumed that the inward migration stops.

In these simulations, if a planetary core reaches 10 Earth masses, it start to accrete a gaseous envelope following the core-instability model (Pollack et al., 1996). The gas accretion is prescribed adding an additional mass to the embryo at each timestep, following the standard (J1) model of Pollack et al., 1996.

In the simulations when the numerical Bulirsch-Stoer part of the integrator is used (i.e., to integrate accurately embryo-embryo or embryoplanetesimal close encounters, close stellar approaches, or the swarm of bodies reaching the inner disk limit), the time step is automatically adapted. 
We use a tolerance of $10^{-12}$. Other effects, as tidal interaction with the central star, quadrupole distortion between the planets, or relativistic effects, where not considered in these set of simulations (see e.g., Trilling et al., 1998, Kiseleva-Egletton and Bois, 2001).

\section{Results}

The combined results of the four simulations are shown in Fig. 1. It is evident that several cores (only one of the planets accrete negligible amounts of gas) reach the inner edge of the simulated disk, where their inward migration stops, and then, they survive with the semi-major axis reported. The migration of the cores is always of type I because their masses are not large enough as to open a gap in the disk. By 10 Myr, when the disk is assumed completely dispersed, several 'hot cores' resembling the ones found around GJ 436 and $\rho$ Cancri are present. During the first stage of the simulations, they growth by the accretion of planetesimals, which is possible for two reasons: first, the fast type I orbital migration, which is shown in Fig. 2, allows the embryos to be continuously immersed in zones not previously depleted of planetesimals 
2 and second, the aerodynamic drag maintains the planetesimals in relatively low excited orbits, a condition needed for efficient accretion. But the most important growth regime is due to mutual accretion between massive embryos during the last stages of the process. This happens because a fraction of them migrated quickly to a narrow zone around the inner edge of the disk favoring mutual collisions and the subsequent formation of Neptune-like planets.

Table 1 shows the final 'hot cores' obteined in each simulation (only planets with $M_{p}>10 M_{\oplus}$ are included) at $t=10 \mathrm{Myr}$. The planets of the simulations Nr2 and Pr1 are remarkably similar to the ones found around GJ 436: $21 M_{\oplus}$ with $a=0.0278$, (Butler et al., 2004); and $\rho$ Cancri: $17.7 M_{\oplus}$ with $a=0.04$, (McArthur et al., 2004). In Fig. 3, we have reproduced the growing process of two planets in the above mentioned simulations. An interesting question arising from the simulations, is the amount of solid mass engulfed by the star. Although it is in general negligible, simulation $\operatorname{Pr} 2$, the only one with two final cores, disperses toward the star about one core mass of solid

\footnotetext{
${ }^{2}$ Note that if the disk is turbulent, the cores can attain a random migration according to Laughlin et al. (2004b) not considered in our model; nevertheless, these fluctuations are largely contained in the random walk introduced by the perturbations between embryos.
} 
material. We believe that our simulations are the first showing this growth regime, that operates on type I migrating protoplanets, in a standard nebular environment. If the new discovered planets formed in a scenario as the one described here, they should be of ice-rock composition with only a thin atmosphere.

\section{Conclusions}

Giant gaseous planets as massive as Jupiter, or even Saturn, were not found in this set of runs, but in all the four simulations, compact systems of two or more Neptune-like planets were produced. Although this result points to the existence of compact planetary systems formed by small giants, it is in contrast, however, to the system orbiting $\rho$ Cancri, which already has other three Jupiter-like planets. This fact could be due to the imposed restriction on the critical core mass, for fast accretion of gas, which nevertheless is not the only possible scenario for the core instability model (Stevenson, 1982). In fact, according to recent results of hydrodynamic accretion of gas around solid cores (D'Angelo et al., 2002), planetary embryos may accrete gas when the solid cores reach only 1-2 Earth masses. 
Our results show that the formation of planets, and systems of Neptunelike planets around solar type stars, as the ones recently discovered, seems to be a natural by-product of planetary formation, and encourage further studies statistically more robust, exploring the whole set of parameters i nvolved in the problem. Planetary migration and the value of the critical core mass for substantial accretion of gas, are crucial factors in determining the distribution of orbits and masses of the formed planetary systems. Taking into account the standard scenario explored here, we predict that a substantial population of 'hot cores' with masses between $\sim 14 M_{\oplus}$ and $\sim 24 M_{\oplus}$ placed at semi-major axis $<0.05 \mathrm{AU}$ await to be discovered in the near future.

Acknowledgements: we acknowledge the finnancial support by IALP, CONICET and AGNPCyT through grant PICT 03-11044 and the anonymous referees that help us to improve the manuscript.

\section{References}

Adachi, I., C. Hayashi and K. I. Nakazawa 1976. The gas drag effect on the elliptical motion of a solid body in the primordial solar nebula. Prog. Theor. 
Phys., 56, 1756-1771.

Beckwith, S. V. W. and A. I. Sargent 1996. Circumstellar disks and the search for neighboring planetary systems. Nature 383, 139-144.

Brunini, A. and M. D Melita 2002. The Existence of a planet beyond 50 AU and the orbital distribution of the classical Edgeworth-Kuiper-Belt objects. Icarus 160, 32-43.

Butler, R. P., Vogt, S. S., Marcy, G. W., Fisher, D. A. Jason, T., Wright, G. W. H., Laughlin, G. and J. J. Lissauer 2004. A Neptune-mass planet orbiting the nearby M Dwarf GJ 436. Astrophys. J. (in press).

Cionco, R. G. and A. Brunini 2002. Orbital Migrations in planetesimal discs: $N$-body simulations and the resonant dynamical friction Mon. Not. Royal. Astron. Soc. 334, 77-86

D'Angelo, G., Kley, W. and T. Henning 2003. Orbital migration and mass accretion of protoplanets in three-dimensional global computations with nested grids. Astrophys. J. 586, 540-561.

Goldreich, P. and S. Tremaine 1980. Disk-satellite interactions. Astrophys. J. 241, 425-441.

Hayashi, C., Nakazawa, K. and Y. Nakagawa 1985. Formation of the solar system. Protostars and planets II, 1100-1153. 
Ida, S. and D. N. C. Lin 2004. Towards a Deterministic Model of Planetary Formation I: a Desert in the Mass and Semi major axis Distribution of Extrasolar Planets Astrophys. J.604, 388-413

Kiseleva-Eggleton L. and E. Bois 2001. Effects of perturbing forces on the orbital stability of planetary systems. Astrophys. J. 553, L73-L76.

Kokubo, E. and S. Ida 1988. Oligarchic growth of protoplanets. Icarus 131, $171-178$.

Kokubo, E. and S. Ida 2000. Formation of protoplanets from planetesimals in the solar nebula. Icarus 143, 15-27.

Laughlin, G., Steinacker, A. and F. Adams 2004a. Type I Planetary Migration with MHD Turbulence Astrophys. J. 608, 489-496

Laughlin, G., Bodenheimer P. and F. Adams 2004b. Core-Accretion Model Predicts Few Jovian-Mass Planets Orbiting Red Dwarfs. Astrophys. J. 612, L73-L76

Lin, D. N. C. and J. Papaloizou 1986. On the tidal interaction between protoplanets and the protoplanetary disk. III - Orbital migration of protoplanets. Astrophys. J. 309, 846-857.

Lin, D. N. C., Bodenheimer, P. and D. C. Richardson 1996. Orbital migration of the planetary companion of 51 Pegasi to its present location. Nature $\mathbf{3 8 0}$, 
606-607.

McArthur, B. E. and 11 colleagues 2004. Detection of a Neptune-mass planet in the $\rho$ Cancri system using the Hobby-Eberley telescope. Astrophys. J. (in press.)

Mizuno, H. 1980. Formation of the giant planets. Progress of Theoretical Physics 64, 544-557.

Pollack, J. B., Hubickyj, O., Bodenheimer, P. and J. J. Lissauer 1996. Formation of the giant planets by concurrent accretion of solids and gas. Icarus 124, 62-85.

Safronov, V. S. 1969. Evolution of the Protoplanetary Cloud and the Formation of the Earth and the Planets, Moscow. Nauka Press.

Stevenson, D. J. 1982. Formation of the giant planets. Plan. Space Sci. 30, 755-764.

Tanaka, H. and S. Ida 1999. Growth of a migrating protoplanet. Icarus 139, 350-366.

Tanaka, H., Takeuchi, T, and W. R. Ward 2002. Three-dimensional interaction between a planet and an isothermal gaseous disk. I. Corotation and Lindblad torques and planet migration. Astrophys. J. 565, 1257-1274.

Tanaka, H. and W. R. Ward 2004. Three-dimensional interaction between a 
planet and an isothermal gaseous disk. II. Eccentricity waves and bending waves Astrophys. J. 602, 388-395.

Thommes, E. W., Duncan, M. J., and H. F. Levison 2003. Oligarchic growth of giant planets. Icarus 161, 431-455.

Trilling, D., Benz, W., Guillot, T., Lunine, J., Hubbard, W. and A. Burrows 1998. Orbital evolution and migration of giant planets: modeling extrasolar planets. Astrophys. J. 500, 428-439

Ward, W. R. 1986. Density waves in the solar nebula - Differential Lindblad torque. Icarus 67, 164-180.

Ward, W. R. 1997a. Protoplanet migration by nebula tides. Icarus 126, 261-281.

Ward, W. R. 1997b. Survival of Planetary Systems Astrophys. J. 482, L211L214. 


\section{Figure captions:}

- Figure 1 Final planetary masses of the embryos as a function of the mean distance to the star. The different symbols represent the planets obtained in each simulation after $10 \mathrm{Myr}$. In the four simulations, planets of 10-24 Earth masses were produced very close to central star.

- Figure 2 Evolution of the semimajor axis of one of the planetary cores in our simulations. The orbital migration obeys to the type I migration, as the theory of disk planet interaction predicts. The migration almost stops when the core reaches the inner edge of the simulated disk. At this location the core suffers strong dynamical interactions with other embryos that also have reached the inner edge of the disk. These interactions produce the observed jumps after $5.5 \mathrm{My}$ at short semimajor axes.

- Figure 3 Mass accretion of two Neptune-like planets vs. the mean distance to the host star. Although the embryos accrete some background planetesimals (small jumps in mass at large semimajor axes), the accretion of substantial amounts of mass is due to the mutual coagulation between embryos, represented by the large steps in mass once 
they reach the inner edge of the disk. The presence of more than one embryo in a narrow zone around the inner edge of the disk produce the jumpy evolution in semimajor axis shown in Fig. 2. 
Table 1: Results of the four simulations at $10 \mathrm{Myr}$ : name of the simulation; total mass, gaseous mass, semi-major axis and eccentricity of the cores obtained; $M_{e n g}$ is the mass of solid material engulfed by the star.

\begin{tabular}{cccccc}
\hline \hline & $M_{\text {core }}\left[M_{\oplus}\right]$ & $M_{\text {gas }}\left[M_{\oplus}\right]$ & $a[\mathrm{AU}]$ & $e$ & $M_{\text {eng }}\left[M_{\oplus}\right]$ \\
\hline \hline $\mathrm{Nr} 1$ & 11.00 & 0.000 & $4.015 \times 10^{-2}$ & $2.495 \times 10^{-2}$ & 0.0 \\
\hline $\mathrm{Nr} 2$ & 23.62 & 0.018 & $3.530 \times 10^{-2}$ & $18.572 \times 10^{-2}$ & 0.0 \\
\hline $\mathrm{Pr} 1$ & 17.90 & 0.000 & $3.840 \times 10^{-2}$ & $0.058 \times 10^{-2}$ & 0.0 \\
\hline $\operatorname{Pr} 2$ & 14.10 & 0.000 & $3.305 \times 10^{-2}$ & $1.393 \times 10^{-2}$ & 0.0 \\
& 14.70 & 0.000 & $4.340 \times 10^{-2}$ & $1.613 \times 10^{-2}$ & 8.3 \\
\hline \hline
\end{tabular}


Figure 1

Nㅣㄴ

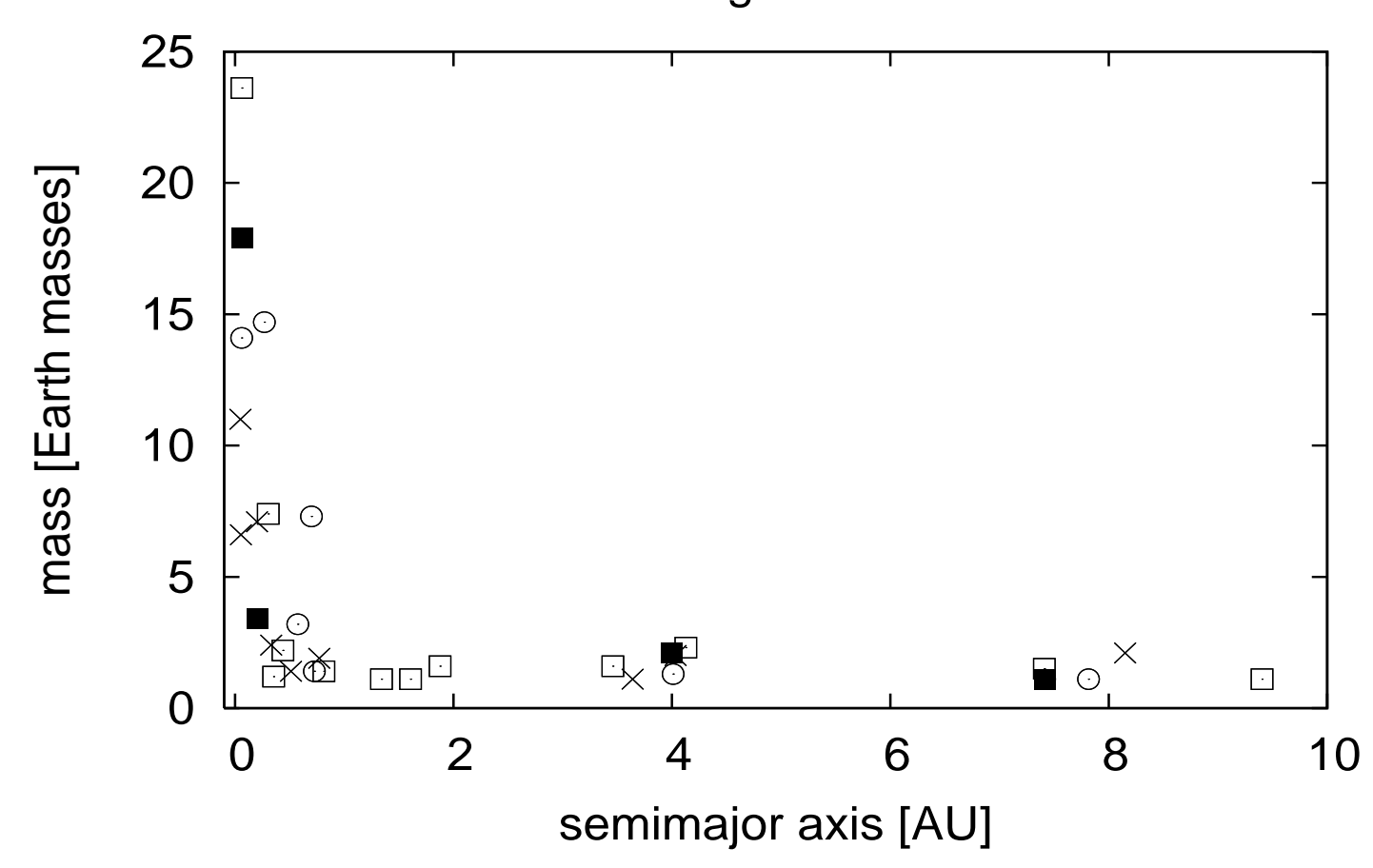


Figure 2

๙ั

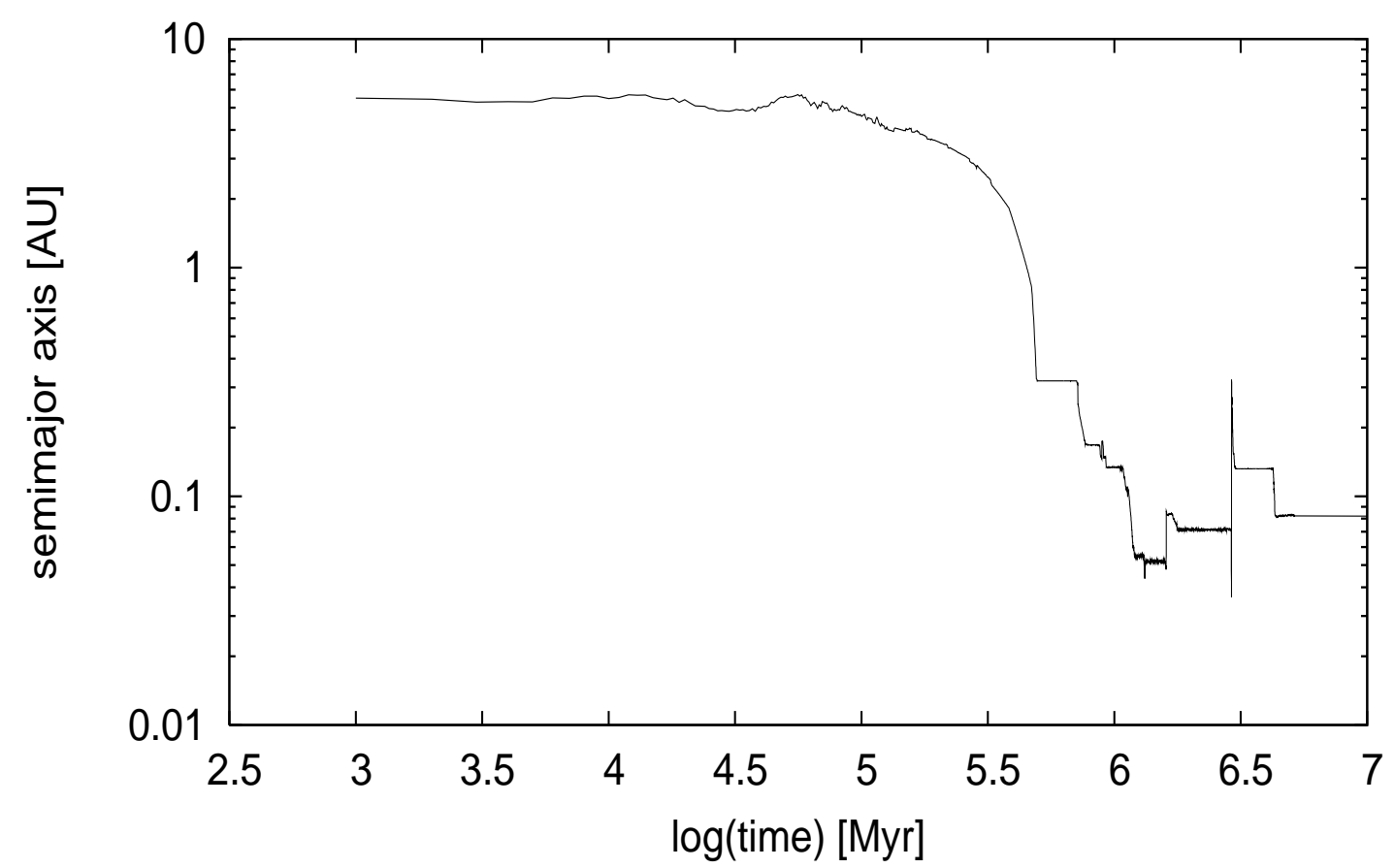


Figure 3

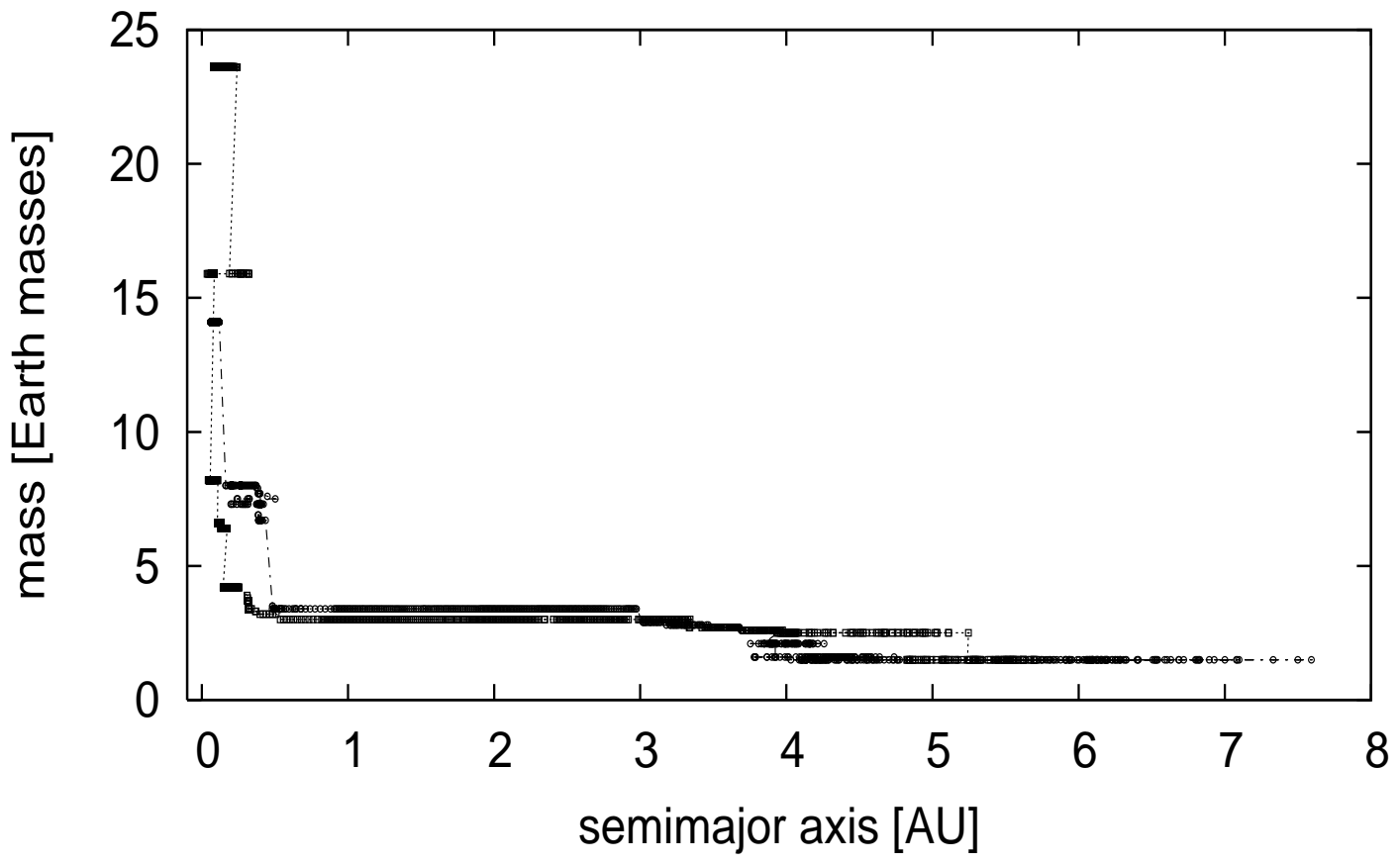

